\title{
Análisis de la Interacción Social en Patologías Mentales por Medios Computacionales: Caso de Estudio Asperger y Trastorno por Déficit de Atención
}

\author{
Manuel A. Arroyave(1), Luis F. Castillo(2,3), Gustavo A. Isaza(2) \\ (1) Universidad de Caldas, Fac. de Ingeniería, Dpto. de Sistemas e Informatica, Manizales, Colombia \\ (2) Universidad de Caldas, Fac. de Ingeniería, Dpto. de Sistemas e Informática, GITIR Grupo \\ Investigación Tecnologías Información y Redes, Manizales, Colombia. \\ (3) Universidad Nacional de Colombia, Sede Manizales, Facultad de Ingeniería y Arquitectura, \\ Departamento de Ingeniería Industrial, GTA en Innovación y Desarrollo Tecnológico, Campus la Nubia, \\ Manizales, Colombia. (e-mail: manuel.arroyavehenao@gmail.com; luis.castillo@ucaldas.edu.co; \\ gustavo.isaza@ucaldas.edu.co)
}

Recibido Jul. 18, 2017; Aceptado Oct. 3, 2017; Versión final Nov. 9, 2017, Publicado Abr. 2018

\begin{abstract}
Resumen
Este artículo presenta el desarrollo de una plataforma computacional para el análisis de interacciones sociales a través de texto en términos de cantidad y contenido de la información con el fin de abordar la intervención y seguimiento de patologías mentales. Algunos índices para medir el desarrollo cognitivo del individuo y técnicas de recuperación de información han sido adaptados al contexto de análisis con el fin de determinar características y encontrar patrones en los componentes lingüísticos y de desarrollo del individuo. Se evaluaron interacciones con diez pacientes, seis diagnosticados con Asperger y cuatro con Trastorno por Déficit de Atención e Hiperactividad. Se encontró evidencia de las características para dichas patologías como cambio abrupto de temáticas y redundancia hacia temas de conversación. La literatura y los resultados sugieren que estos sistemas para comunicación mediada por computador permiten el descubrimiento de información a la vez que potencializan el desarrollo cognitivo del individuo.
\end{abstract}

\section{Social Interaction Analysis in Mental Pathologies by Computational Means: Study Case Asperger and Attention Deficit Disorder}

\begin{abstract}
This paper presents the development of a computational platform for social interaction analysis through text in terms of quantity and content of information to approach the intervention and tracing of mental pathologies. Some indexes to measure the individual cognitive development and information retrieval techniques has been adapted to the analysis context aimed to determine characteristics and find patterns on the individual linguistic components and his development. The study considered ten patients: six diagnosed with Asperger Syndrome and four with Attention Deficit Hyperactive Disorder. Evidences of the characteristics for each pathology such as abrupt change of a conversation matter, and redundancy over conversation subjects were found. Literature results and the results of this study suggest that these systems for computed mediated communication allow discovering information and empowering the cognitive development of a person.
\end{abstract}

Keywords: mental pathology; cognition; social interaction; Asperger; Attention Deficit Hyperactive Disorder 


\section{INTRODUCCIÒN}

A nivel de interacción social las personas que han sido diagnosticadas con alguna patología mental experimentan desafíos en su vida diaria que afecta de forma significativa la calidad de vida de las mismas (Boucenna et al., 2014). Las condiciones mentales comprenden los procesos de aprendizaje, razonamiento, percepción, atención, memoria (Fernández-López et al., 2013), comportamiento, resolución de problemas, toma de decisiones y procesamiento del lenguaje, siendo este último de vital importancia para una correcta integración e interacción en sociedad, lo que permite a las personas desarrollarse de manera óptima como individuos (Davis-McShan, 2015). Las TICS se han posicionado como medio para fortalecer los procesos cognitivos a través de herramientas y medios de aprendizaje que facilitan la formación de los individuo (Narvaez-Romo et al., 2017), pues debido a la complejidad, variedad y grado de las diferentes condiciones mentales, algunos métodos tradicionales de intervención no se presenten como los adecuados para la correcta evolución del paciente. Condiciones como el autismo en donde la confrontación cara a cara con otras personas puede resultar estresante para el individuo (Benford, 2008), o el Trastorno por Déficit de Atención e Hiperactividad donde las características propias de los individuos pueden dificultar la interacción (Uekermann et al., 2010), pueden presentar resultados lentos o adversos cuando son tratados bajo los parámetros tradicionales de intervención. El enfoque metodológico presente en disciplinas como la psicología cognitiva, la psicolingüística y la neuropsicología mantienen su foco de interés en una serie de principios y descripciones teóricas en las cuales basa sus intervenciones con pacientes, acompañados de un conjunto de procedimientos de análisis experimentales ajustados a dichos planteamientos. Aunque de manera general los resultados son optimistas, son tratamientos que requieren, tiempo, espacio (Mohr et al. 2013) y recursos económicos, limitando el acceso a estos programas para una gran parte de la población (Paul et al., 2016).

Diferentes estudios han demostrado le relación entre patologías mentales y el desarrollo del lenguaje (Stephanie y Julie, 2015). De igual forma variadas herramientas han sido implementadas con el fin de asistir a los pacientes bajo alguna condición mental con los requerimientos de sus necesidades comunicativas como en el caso de las Comunicaciones Aumentadas y Colaborativas (ACC) (Campigotto et al., 2013; FernándezLópez et al., 2013), que corresponden a sistemas generalmente pictográficos por medio de los cuales el individuo puede hacer conocer sus necesidades. Sin embargo, estas no trascienden en la intervención y aunque apoyan de manera significativa la comunicación con el paciente, no reflejan una recuperación que aumente con el tiempo en los canales comunicativos básicos del individuo (habla, lectura, escritura). En igual medida, los resultados observables de estas investigaciones son obtenidos a través de la abstracción del paciente con respecto al uso de las tecnologías para por medios offline calificar y evaluar el uso de la herramienta; esto es, a través de la realización de encuestas, cuestionarios y análisis de datos post-facto a la interacción (Campigotto et al., 2013; Davis-McShan, 2015) lo que puede no reflejar de forma real los resultados en la interacción con otras personas a través de las plataformas.

En algunos trabajos se evidencian procedimientos que se realizan de forma manual para recolectar la información (Beykikhoshk y Caelli, 2014; Davis-McShan, 2015; Locke et al., 2014) y ajustarla a formatos que puedan ser procesables por un sistema computacional (Tanaka et al., 2014). Estos procesos requieren tiempo (Rouhizadeh, 2015), por lo que no pueden ser realizados y analizados durante el proceso mismo de interacción con el paciente, lo que limita el seguimiento constante y real sobre la evolución diaria de la persona. Otros estudios se basan en recolectar información generalizada sobre una condición disponible en la web (Beykikhoshk y Caelli, 2014; Paul et al., 2016; Sundaram et al., 2012). Allí, no se establece un seguimiento personalizado a un individuo o grupo de estos y como en muchos estudios se promedian medidas que, aunque pueden aplicar para características generales de la población y generalmente confirman los resultados ya obtenidos, no ofrecen resultados diferenciales.

Diferentes estudios se han llevado a cabo con el fin de extraer información relacionada a variadas patologías mentales de diversas plataformas para comunicación en línea como Twitter, Facebook (Conway y O'Connor, 2016) y sistemas CMC como mensajería instantánea, SMS (Rana et al., 2015), correo electrónico, salas de chat publicas entre otros. En plataformas como Twitter se han centrado variados esfuerzos (Beykikhoshk y Caelli, 2014) para la aplicación de técnicas computacionales orientadas a la recuperación de información con relación a diferentes condiciones patológicas. Conteo promedio de palabras por tweet y técnicas asociadas a frecuencia de los términos fueron las técnicas aplicadas para encontrar características en los tweets relacionados. De manera similar, en (Conway y O'Connor, 2016; Paul et al., 2016) se usa Twitter como fuente de datos para recuperación de información que ayude al monitoreo y vigilancia de diferentes condiciones patológicas de forma general sobre la población.

En los trabajos de Rouhizadeh (2015), Rouhizadeh et al., (2014), Regneri y King, (2014) estudios fueron llevados a cabo evaluando diversas situaciones como temáticas o conceptos fuera de tópico para una situación planteada, temas repetitivos de conversación, interés restringidos en diálogos y narraciones; en los que se encontró que se puede tener una mejor interacción cuando se permite al individuo bajo la condición expresarse 
sobre sus temas constantes de interés, adicional a que se confirman diversos resultados sobre la utilización de términos y conceptos inesperados utilizando el índice tfidf. De igual forma se evaluó la similitud entre narrativas de individuos con autismo y el grupo de control, encontrando diferencias en los estilos de ambas fuentes. Tanaka et al., (2014) realiza un trabajo similar usando conteo de palabras y tfidf. De igual forma en el estudio de Saint-Georges et al., (2011) se aplican métodos de IR como tfidf y similitud para análisis con bigramas teniendo como fuente de datos interacciones captadas en video entre niños con autismo y sus padres, las cuales son categorizadas de forma manual con respecto a la identificación de cierto evento de interacción en un video. Davis-McShan, (2015) evalúa el uso de las CMC a partir del tiempo que los usuarios invierten en las plataformas. Burke et al. (2010); van der Aa et al., (2016) evaluaron la satisfacción de adultos con autismo con respecto al uso de CMC a través de entrevistas y cuestionarios.

Teniendo en cuenta lo anterior se evidencia la necesidad de analizar los datos resultantes de interacciones sociales en medios donde estas se pueden ver potencializadas debido a las dificultades cognitivas que presentan los individuos cuando la interacción es más física y presencial. Un análisis de este tipo permite un acercamiento diferente a la evaluación de resultados los cuales se dan en base a la propia interacción del paciente en las plataformas y no en base a información obtenida en tareas post-facto como con el uso de cuestionarios o recolección, alistamiento y procesamiento de la información, lo que requiere tiempo y recursos adicionales. Nuevas metodologías deben plantear el análisis de resultados desde diferentes contextos, y es evidente como la modificación de estos en áreas del aprendizaje pueden ayudar a mejorar los métodos tradicionales de acompañamiento e intervención (Huapaya et al., 2005) con el apoyo de las tic (NarvaezRomo et al., 2017). Para lo anterior se proporciona una plataforma para intercambio de mensajes que permite el procesamiento de los mismos a medida que son generados o cargados en el sistema. Para este procesamiento se han adaptado técnicas usadas en entornos para Recuperación de Información e índices orientados a determinar la cantidad de información, así como también se proporciona una interfaz de visualización en la cual los resultados pueden ser analizados mediante diferentes gráficos.

\section{MATERIALES Y MÉTODOS}

Se ha implementado una plataforma computacional accesible a través de la web y en pruebas sobre dispositivos móviles sobre la cual se pueden establecer canales de comunicación a nivel textual entre usuarios registrados. El sistema guarda registro de las conversaciones para su análisis y de igual forma cuenta con la opción de cargar archivos con transcripciones de conversaciones y producciones textuales. Diferentes medidas han sido adaptadas para identificar algunas características de la comunicación como cantidad y tamaño de los mensajes, determinar la función de los términos utilizados y correlacionar las interacciones en base a estos y su relevancia para cada interacción. Los resultados han sido plasmados de forma gráfica para agilizar el análisis de los datos e interpretación de los mismos.

\section{Roles y Requerimientos}

Para pacientes y tutores es necesario que estos tengan habilidades básicas de lectura y escritura, así como también las habilidades motrices necesarias para realizar estas actividades ya sea por medio de la digitación del texto en un teclado o pantalla de dispositivo móvil. La opción de análisis se puede activar para los usuarios tutores que serán los encargados de la interpretación de resultados.

\section{Arquitectura}

Se precisó el uso de un servidor de mensajería para la gestión de usuarios y el intercambio de mensajes en la plataforma. Para tal fin, Openfire, un servidor de código abierto bajo la licencia apache y que funciona en base al protocolo abierto para mensajería XMPP se eligió como la mejor opción debido a la completitud del mismo en cuanto a administración y gestión, facilidad de integración con sistemas en otros lenguajes y los recursos disponibles para ampliar y adaptar sus capacidades. El componente interactivo y que está en directo contacto con el cliente ha sido implementado en Phonegap, un marco de trabajo para desarrollo de aplicaciones móviles que de igual forma permite compatibilidad con navegadores web de escritorio y compilación de los sistemas para diferentes plataformas como Android e iOS.

Esta plataforma funciona bajo el estándar html5 con soporte a lenguajes de scripting JavaScript y etiquetado CSS. El componente grafico se compone de una librería JavaScript denominada d3.js que presenta buena integración con el modelo de despliegue de Phonegap. La comunicación entre Openfire y Phonegap se da por un componente externo denominado Strophe.js, el cual contiene las herramientas necesarias para inicio de sesión, indicación de estado, despliegue de contactos e intercambio de mensajes. El componente de análisis está desarrollado enteramente en java y comprende un servicio web operando bajo un servidor Glassfish. El servicio java contiene la implementación de los índices y modelos para el análisis de los datos teniendo en cuenta que estos pueden venir desde un archivo plano cargado al sistema o desde la información almacenada 
en Base de Datos. La persistencia del sistema se encuentra bajo un motor de base de datos Mysql y allí se conectan tanto el servidor de mensajería y sus componentes extras, así como también el servicio de procesamiento para extracción de información y consecuente generación de resultados. El despliegue físico de los componentes se muestra en la Figura 1.

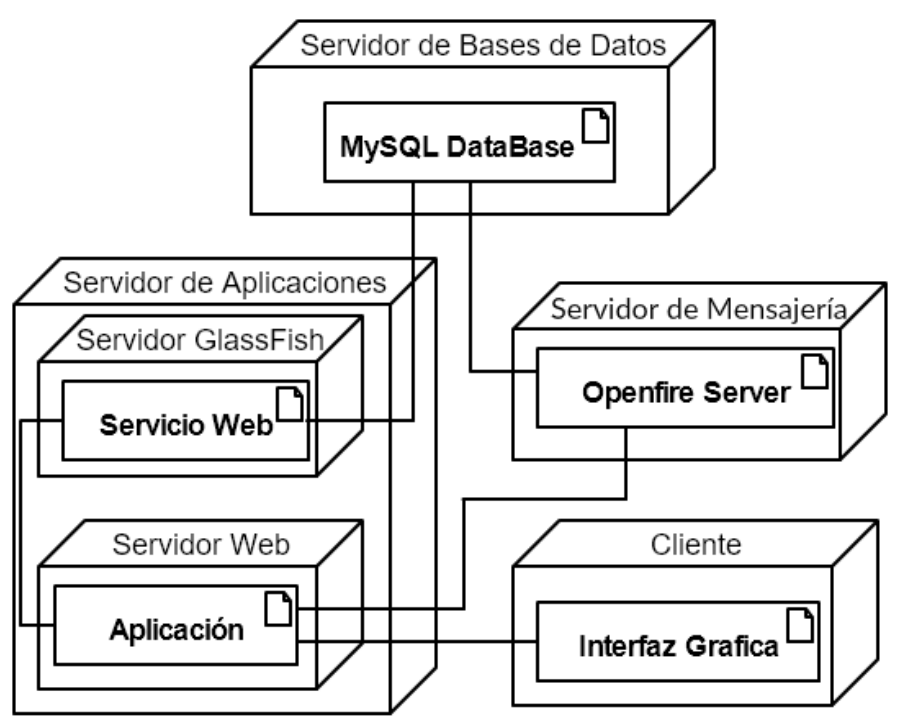

Fig. 1: Despliegue físico de componentes

\section{Pre Procesamiento}

En el contexto aplicado es recomendable realizar una normalización de los textos (Rouhizadeh, 2015) con el fin de optimizar el rendimiento del procesamiento y el proceso de análisis. La remoción de palabras sin contenido o stopwords como artículos y pronombres, permite acotar los términos de análisis a aquellos con mayor contenido y significancia, estableciendo relevancia a palabras más complejas. De igual forma una lematización; que consiste en una reducción de los signos a su raíz, permite una agrupación de los mismos brindando la posibilidad de reducir los costos de análisis mejorando el mismo en cuanto a la utilización y relación de los símbolos lingüísticos pues reduce significativamente la dimensionalidad para el caso del modelo generado en el espacio vectorial de documentos.

\section{Distribución Ordenada y Longitud Media del Enunciado en Palabras LMP}

La LMP es un índice usado para medir el desarrollo del lenguaje especialmente en niños, pues indica que a mayor número de palabras las construcciones son más complejas (Ayala Flores, 1988). Este se mide en base a la cantidad de términos encontrados en un enunciado, siendo este último entendido como el contenido entre pausas en un escrito o narración. Para determinar la cantidad de información presente en la interacción se ha diseñado una distribución ordenada en la cual se comparan número de líneas o mensajes por sesión de comunicación, adicional a que se ha adaptado el índice LME para determinar la longitud media de la comunicación por palabras. El resultado de lo anterior es plasmado en un gráfico de línea como en la Figura 2 , en el cual el eje " $x$ " representa las comunicaciones de la interacción y el eje "y" el número de mensajes por cada una de estas. El índice LMP se calcula en base al número de palabras en la comunicación y/o párrafo, valor que es dividido por el número de comunicaciones y/o párrafos presentes en la interacción y/o escrito.

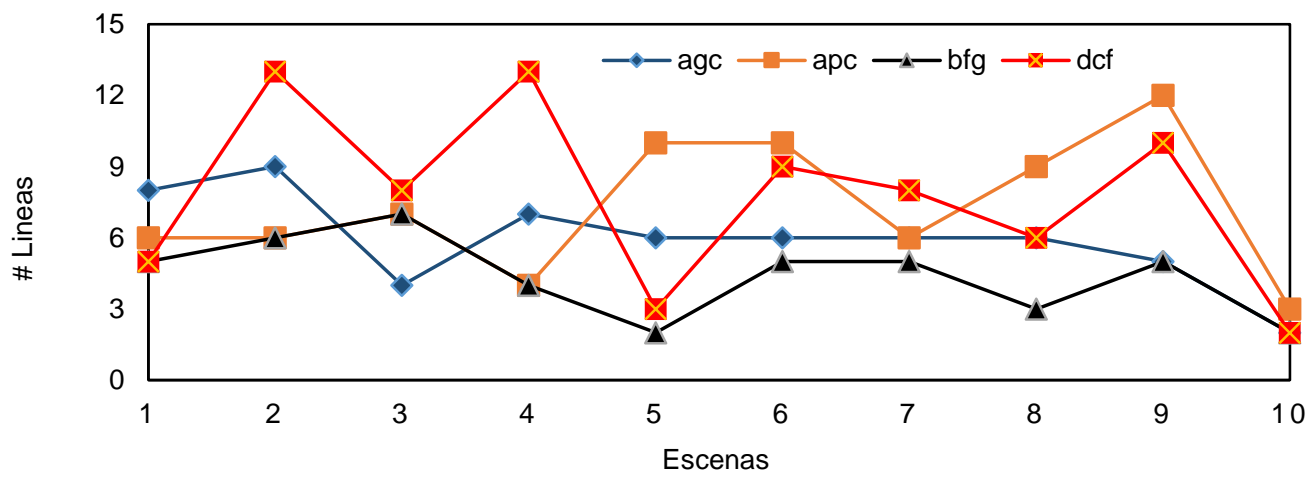

Fig. 2: Longitud Media del Enunciado y Distribución Ordenada que compara comunicaciones y número de mensajes 
En (1), LMPi indica la longitud de la comunicación $\mathrm{i}$ en palabras, $\Sigma x \in$ i representa la cantidad de palabras $\mathrm{x}$ en la comunicación i y $\sum$ i es la cantidad de comunicaciones en la interacción.

$$
\mathrm{LMPi}=\frac{\sum x \in i}{\sum i}
$$

La representación gráfica seleccionada permite evaluar el desarrollo de la interacción en el tiempo en base a la cantidad de información para cada intercambio. De esta forma es posible determinar las reacciones a diferentes estímulos con la posibilidad de realizar una visualización comparativa de las interacciones que el especialista ha tenido con diferentes pacientes, dando la posibilidad de evaluar por ejemplo el desempeño de los individuos bajo un programa de intervención específico.

\section{Frecuencia del Termino Frecuencia Inversa del Documento TFIDF}

La frecuencia de los términos utilizados en contraste con la frecuencia inversa del documento se expone como una de las medidas más usadas de Recuperación de Información (Rouhizadeh, 2015). El índice permite identificar los términos que son más relevantes para un escrito en comparación con los términos encontrados en otros escritos (Regneri y King, 2014). Para el proceso comunicativo de estudio, se adaptó el análisis para los términos en una sesión de comunicación en referencia a las demás comunicaciones de la interacción. El índice TFIDF es calculado en diversos pasos:

Frecuencia del Termino TF: Se extrae el número de veces que el término aparece en la porción de texto analizado y se divide por el número de palabras en dicho texto. En (2), $t f(t, d)$ indica la frecuencia del termino t, en la comunicación d; que es igual a la cantidad de veces que el termino t se encuentra en la comunicación d, dividido por el total de términos w presentes en la misma comunicación d (Manning et al., 2009; Yamamoto y Takagi, 2007).

IDF: La frecuencia inversa del documento se calcula en base al número de comunicaciones que contienen el signo analizado. Para esto, se obtiene la cantidad total de comunicaciones en la interacción analizada y se divide por el número de comunicaciones que contienen el término en análisis. Finalmente se toma el logaritmo de dicho cociente. En (3), $\mathrm{N}$ representa el total de comunicaciones en la interacción y dfi, el número de documentos que contienen el término $t$ (Yamamoto y Takagi, 2007).

TFIDF: Se obtiene mediante el producto de los resultados (4) para los índices anteriores (Saint-Georges et al., 2011).

$$
\begin{aligned}
& \mathrm{tf}(\mathrm{t}, \mathrm{d})=\sum(\mathrm{t}, \mathrm{d}) / \sum(\mathrm{w}, \mathrm{d}) \\
& i d f_{i}=\log \frac{N}{d f_{i}} \\
& t f i d f(t, d)=t f(t, d) \times i d f_{i}
\end{aligned}
$$

\section{Modelo de Espacio Vectorial}

Este modelo ampliamente utilizado para tareas de Recuperación de Información permite representar los documentos como vectores según el peso asignado para cada uno de sus términos (Manning et al., 2009). El resultado del modelo es una matriz de $n^{\star} m$ donde $n$ es la cantidad de palabras presentes durante toda la interacción y $\mathrm{m}$ la cantidad de comunicaciones que conforman la misma. De esta forma para cada intersección se tiene el peso tfidf calculado para la palabra en la comunicación analizada, asignando valores de 0 cuando el termino no se encuentra en la comunicación (Hassanpour et al., 2013).

\section{Similitud Coseno}

Es una medida de similitud que se basa en el coseno del ángulo entre dos vectores de documentos para determinar la correlación entre los mismos (Huang et al., 2012). Si los vectores son iguales el ángulo es 0 y el coseno 1, mientras que si los vectores son ortogonales el coseno estará más próximo a 0 . De forma general, la similitud coseno se calcula mediante el producto punto entre los vectores que representan los documentos a comparar, dividido por el producto de las longitudes Euclidianas de ambos vectores (Hassanpour et al., 2013; Manning et al., 2009).

$$
\operatorname{Sim} \operatorname{Cos}(d 1, d 2)=\overrightarrow{d 1} \cdot \overrightarrow{d 2} /(\|\overrightarrow{d 1}\| \times\|\overrightarrow{d 2}\|)
$$


En (5) SimCos $(\mathrm{d} 1, \mathrm{~d} 2)$ indica la similitud entre los documentos $\mathrm{d} 1 \mathrm{y} \mathrm{d} 2$ representados como vectores. La expresión $\mathrm{d} 1$. d2 es el producto punto entre los vectores comparados y el producto entre las longitudes Euclidianas de ambos vectores está representado por el miembro entre paréntesis.

Una forma de representar lo anterior de forma gráfica es a través de un grafo no dirigido como en la Figura 3 en la cual los nodos representan las diferentes comunicaciones de la interacción y las aristas indican si existe relación y el grado de las mismas. Para el caso de estudio y teniendo en cuenta la naturaleza de los datos evaluados, se han caracterizado los nodos por un color distintivo en la aplicación para cada grupo de comunicaciones en la interacción. Se ha definido un umbral para determinar cuando el valor indicado por el cálculo de la similitud indica relación entre las partes, creando una arista entre los nodos cuando se supera este umbral e indicando el grosor de la misma el grado de la relación. De esta forma es posible visualizar tópicos de conversación, términos y palabras usadas de forma más relevante, relaciones entre conceptos y conversaciones y para cada situación particular la evolución en la capacidad comunicativa del individuo analizado.

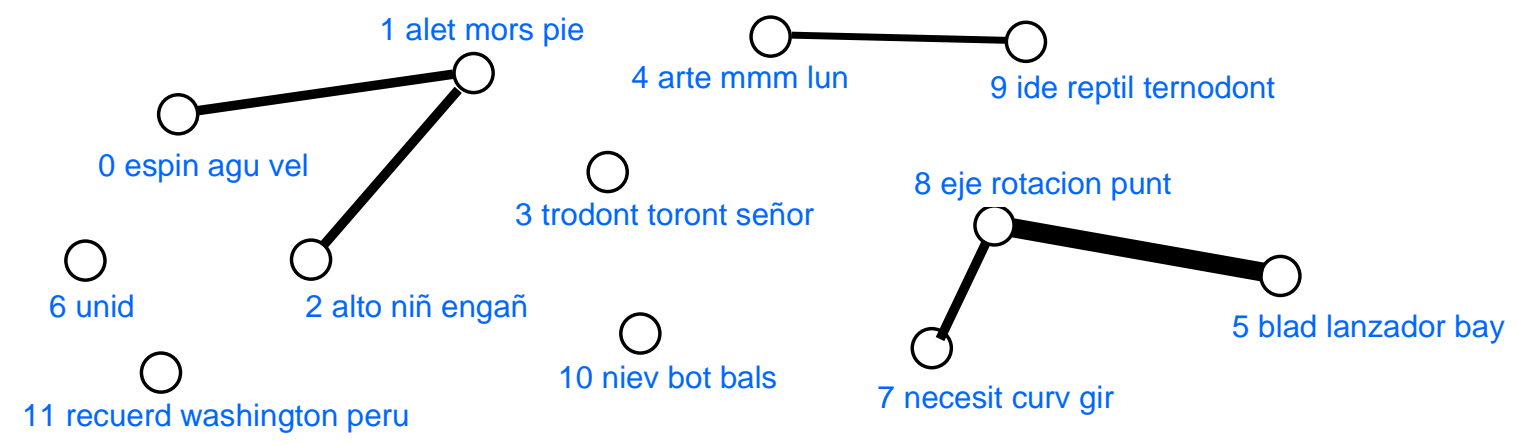

Fig. 3: Similitud Coseno Antonio.

Para evaluar el sistema en términos de funcionalidad, se usaron recursos disponibles en la web como el Corpus Perla CP y algunas transcripciones de conversaciones en estudios relacionados. Los recursos accedidos del CP comprenden los Trastornos por Déficit de Atención y/o Hiperactividad TDAH y Asperger, adicional a 2 conversaciones con pacientes diagnosticados con Asperger pertenecientes al estudio llevado a cabo por Paola Verano (Verano, 2013). Para nuestro estudio, se han cargado y analizado 4 conversaciones de corpus perla relacionadas a Asperger, así como también las 2 conversaciones indicadas anteriormente. Las conversaciones identificadas en el corpus perla para el síndrome de asperger siguen un patrón establecido en el que el entrevistador estructura una interacción en base a unas preguntas al paciente por lo que es posible comparar las diferentes interacciones y evaluar las reacciones a diferentes estímulos que en este caso se representan como preguntas relacionadas a escenas mostradas en distintas diapositivas, adicional a conversaciones tradicionales donde se indagan los gustos e intereses del paciente. Por otro lado, las conversaciones con pacientes de Asperger llevadas a cabo por verano muestran una interacción sin estructura cuyo desarrollo se da en base a diálogos sobre los gustos u otros tópicos de la vida general del paciente.

\section{EVALUACIÓN Y RESULTADOS}

La figura 2 refleja las cuatro interacciones con los 4 pacientes de Asperger en términos de número de líneas por escena, lo que nos permite evidenciar los siguientes momentos de las interacciones: Con el paciente denominado agc y en contraste con el contenido del dialogo se evidencian respuestas concretas y coherentes a las preguntas sin querer explicar o complementarlas con contenidos adicionales; lo que en la figura se puede evidenciar pues representa cierta linealidad en la interacción con un número de líneas medio por escena con respecto al resto de interacciones por lo que sirve de punto de referencia para los demás análisis, ya que según el contenido de la misma se clasifica como una conversación normal. Analizando la interacción con el paciente dcf, se observa como al inicio de la conversación se dispersa y complementa las respuestas con sucesos pasados de la vida real, adicional a que describe lo de las imágenes mostradas sin antes haber realizado la pregunta; lo que implica un mayor número de líneas para las interacciones iniciales de la conversación. Aunque inicia de forma apresurada queriendo adelantarse a los hechos, una vez se toma el control de la interacción el niño responde de forma concreta y con aserciones coherentes respecto a los hechos mostrados, generando inclusive conclusiones en base a detalles de las imágenes. De forma paralela esto se puede observar en el análisis grafico pues inicialmente se observan unos picos en la interacción, acoplándose un poco más a la 
interacción de referencia a medida que esta se desarrolla sin embargo estando siempre el cálculo de los resultados por encima de lo que hemos considerado como de referencia (líneas que en la gráfica son las más inferiores), lo que indica que el paciente puede ser un poco más intenso o extrovertido en su actuar o en su expresión.

La figura 2 refleja como dos interacciones en especial presentan un mayor contenido siendo estas identificadas para pacientes que tienden a una mayor dispersión en sus procesos comunicativos, aunque de igual forma se observa que cuando responden a las preguntas se extienden con complementos o explicaciones que denotan algún grado de imaginación, extroversión o acceso a recuerdos que en general pueden ser tomados como aspectos positivos para el desarrollo del individuo. Se nota como un proceso simple de llamado de atención puede ayudar a que los procesos comunicativos del individuo se centren en la acción propia y en el desarrollo de la interacción. Por otro lado, las representaciones más inferiores de la gráfica representan interacciones con respuestas concretas a los estímulos (preguntas en base a diapositivas), por lo que la comunicación se facilita con dichos individuos sin necesidad de acciones correctivas para el desarrollo de la misma.

Una observación importante se refleja en cuanto al retorno abrupto a tópicos de interés por parte de los pacientes diagnosticados con trastorno del espectro autista para este caso con síndrome de asperger. En las conclusiones del estudio llevado a cabo por Verano y del cual hacen parte 2 de los conjuntos de datos analizados se identifican situaciones en ambas interacciones que son percibidas como retorno a temas de interés de los pacientes cuando se interactúan con estos, más sin embargo en las transcripciones se evidencia que para el caso de Álvaro se inicia el desarrollo de un tema en específico; como en el caso del concurso gastronómico observado por televisión; y a medida que el paciente describe su experiencia el entrevistador intentar cambiar o introducir un nuevo tema sin que el paciente haya terminado de dar su descripción sobre la situación. Por otra parte, y en relación con lo observado en la comunicación con Antonio son más evidentes los cambios de tema retornando a tópicos de interés propios de los pacientes, ya que, aunque por ejemplo al inicio de la conversación se dialoga sobre dinosaurios, cuando el entrevistador intenta dialogar sobre otros tópicos el paciente sugiere que lo que le gusta o recuerda son los dinosaurios.

Esta situación es analizada por nuestro sistema a través del índice de similitud el cual nos permite identificar cuando y en qué grado diferentes porciones de la interacción pueden estar relacionadas entre sí. Para el caso de Antonio los resultados gráficos en la Figura 3 muestran una serie de relaciones entre diferentes escenas 0 interacciones de la comunicación, lo que indica que en algún momento durante una interacción se tocaron temas o se hizo uso de términos presentes en otras interacciones, mientras que para el análisis de la interacción con Álvaro no se evidencia relación de las interacciones, por lo que se supone la interacción fue más fluida desarrollando los temas que se introducían en el momento. Lo anterior se alinea con los resultados encontrados por Verano donde se sugiere que "el niño con "más Asperger" (Antonio) es el que presenta Unidades incoherentes que retornan abruptamente al tópico favorito. Sin embargo, el otro niño (Álvaro) no genera ninguna unidad de este tipo durante la conversación. Por lo tanto, en este caso, se puede concluir que el niño que está más cercano al Autismo de Alto Funcionamiento también posee más dificultades durante la interacción verbal que mantiene conmigo." (Verano, 2013).

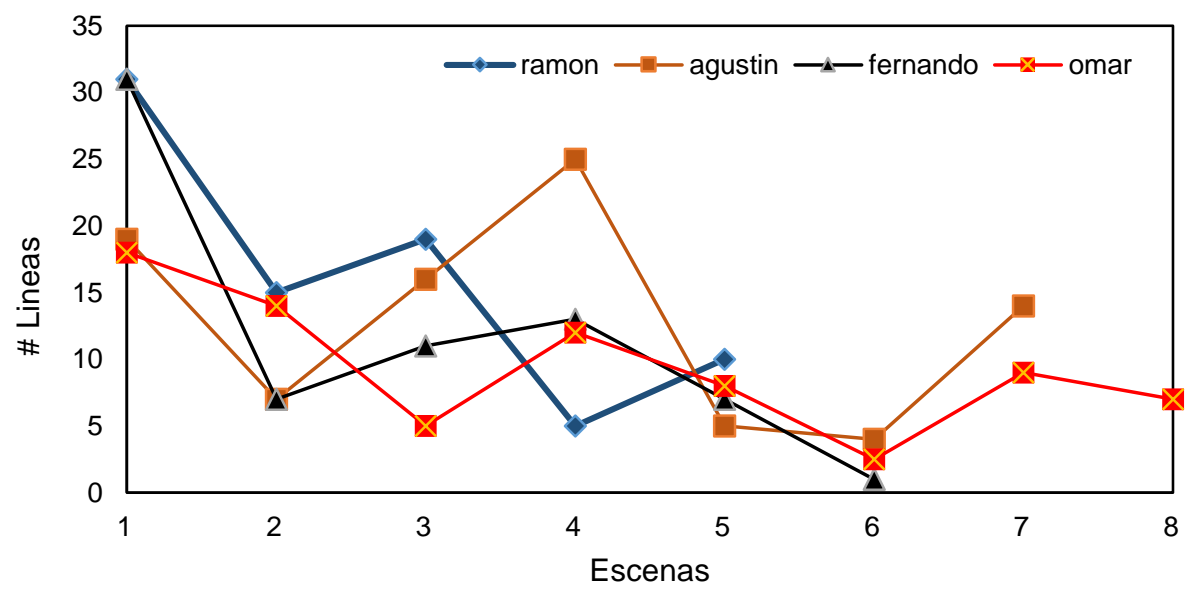

Fig. 4: Distribución Ordenada TDAH

De forma complementaria los resultados para TDAH en la Figura 4 reflejan que a medida que se desarrolla la interacción, y debido a las intervenciones constantes del especialista, la comunicación decrece en número de líneas para todas las intervenciones conduciendo a que las repuestas de los pacientes sean más concretas y precisas. De igual forma se evidencias picos en algunas situaciones que permiten identificar ciertas características de los individuos que han sido evaluados bajo esta patología. 


\section{DISCUSIÓN FINAL}

Adicional a los beneficios reportados por los diferentes estudios revisados en cuanto al uso de tecnologías como las $\mathrm{CMC}$ en la intervención para diferentes estados de patologías mentales, se observa que los resultados obtenidos referentes al análisis de interacciones sociales a través de medios virtuales basados en texto representan una fuente de información importante para terapeutas, especialistas y familiares tanto para la intervención como para el acompañamiento. Debido a que la intervención temprana en este tipo de situación representa una ventaja con respecto a la evolución posterior del individuo (Green et al., 2010), información relevante sobre las interacciones y como estas se están desarrollando en el tiempo a medida que ocurren y avanzan, resultan un factor determinante para la toma de decisiones en pro de la recuperación y rehabilitación del paciente.

El tipo de tecnología planteada permite establecer comunicación en diferentes momentos del día sin requerir de una interacción cara a cara entre los individuos, lo que permite ampliar los rangos de intervención causando mayor producción de datos cuyo análisis continuo aporta en el establecimiento de estrategias óptimamente adaptadas para el tratamiento de individuos. Así mismo la carga de interacciones pre procesadas es de gran ayuda para complementar los análisis de datos referentes a interacciones y desarrollo social del individuo teniendo en cuenta los corpus existentes o historias clínicas que puedan tener actualmente los especialistas que tratan las diferentes condiciones mentales. La identificación de patrones por medios gráficos permite reducir los tiempos de análisis y generar conclusiones de forma más óptima, lo que permite identificar estrategias para ser aplicadas en el corto plazo para aportar en el desarrollo general de los pacientes. De igual forma la implementación de algoritmos cuyo procesamiento se da sobre texto pueden ayudar a la identificación de condiciones características de individuos cuando estos se analizan a través de los textos en sus interacciones o producciones textuales.

\section{CONCLUSIONES}

A partir del análisis y los diferentes resultados es posible llegar a las siguientes conclusiones: 1) la adaptación de técnicas de IR como TFIDF en contextos de interacción sobre patologías mentales es aplicable para la identificación de patrones característicos en las mismas; para este caso específico en condiciones autistas y TDAH; 2) no se reportaron evidencias de sintomatologías del espectro autista en los resultados obtenidos para TDAH en lo referente a recurrencia a temas de interés, lo que permite mejorar el conocimiento en cuanto a la relación reportada históricamente entre estas patologías y 3) las visualizaciones de las interacciones según diferentes propósitos de análisis permiten inferir características de la comunicación y de los individuos que interactúan y que no son evidentes durante el acto mismo de interacción. La visualización de la información permite el descubrimiento de relaciones ocultas entre los datos mejorando los tiempos de análisis e intervención.

\section{REFERENCIAS}

Ayala Flores, C. L., Evaluación de la sintaxis durante el desarrollo del lenguaje, https://goo.gl/qsqJGm, ISSN: 0214-283x, Parole: revista de creación literaria y de filología, 1(1), 127-138 (1988)

Benford, P., The use of Internet-based communication by people with autism, Tesis Doctoral, Dpto. Psicología, University of Nottingham, Nottingham, Inglaterra (2008)

Beykikhoshk, A., y Caelli, T., Data-Mining Twitter and the Autism Spectrum Disorder: A Pilot Study, International Conference on Advances in Social Networks Analysis and Mining (ASONAM 2014) Data-Mining, 349-356, Beijing, China, 17 al 20 de Agosto (2014)

Boucenna, S., Narzisi, A., Tilmont, E., Muratori, F., Pioggia, G., Cohen, D., y Chetouani, M., Interactive Technologies for Autistic Children: A Review, doi: 10.1007/s12559-014-9276-x, Cognitive Computation, 6(4), 722-740 (2014)

Burke, M., Kraut, R., y Williams, D., Social use of computer-mediated communication by adults on the autism spectrum, doi: 10.1145/1718918.1718991, Proceedings of the 2010 ACM conference on Computer supported cooperative work CSCW '10, $425-434$ (2010)

Campigotto, R., McEwen, R., y Demmans Epp, C., Especially social: Exploring the use of an iOS application in special needs classrooms, doi: 10.1016/j.compedu.2012.08.002, Computers and Education, 60(1), 74-86 (2013)

Clarke, G., y Yarborough, B. J., Evaluating the promise of health IT to enhance/expand the reach of mental health services, doi: 10.1016/j.genhosppsych.2013.03.013, General Hospital Psychiatry, 35(4), 339-344 (2013)

Conway, M., y O'Connor, D., Social Media, Big Data, and Mental Health: Current Advances and Ethical Implications, doi: 10.1016/j.copsyc.2016.01.004, Current Opinion in Psychology, 9, 77-82 (2016)

Davis-McShan, M.L., Impact of Computer-Mediated Communication Duration on Adolescent Social Self-E cacy, Social Anxiety, and Depression, Tesis Doctoral, Dpto. Psicologia, Walden University, Minessota, USA (2015) 
Fernández-López, Á., Rodríguez-Fórtiz, M.J., Rodríguez-Almendros, M. L., y Martínez-Segura, M. J., Mobile learning technology based on iOS devices to support students with special education needs, doi: 10.1016/j.compedu.2012.09.014, Computers and Education, 61(1), 77-90 (2013)

Green, J., Charman, T., McConachie, H., Aldred, C., Slonims, V., Howlin, P., Consortium, P., Parent-mediated communication-focused treatment in children with autism (PACT): a randomised controlled trial, doi: 10.1016/S01406736(10)60587-9, Lancet, 375(9732), 2152-2160 (2010)

Hassanpour, S., O'Connor, M. J., y Das, A. K., A semantic-based method for extracting concept definitions from scientific publications: evaluation in the autism phenotype domain, doi: 10.1186/2041-1480-4-14, Journal of biomedical semantics, 4(1), 4 - 14 (2013)

Huang, L., Milne, D., Frank, E., y Witten, I. H., Learning a Concept-based Document Similarity Measure, doi: 10.1002/asi.22689, Journal of the American Society for Information Science and Technology, 63(8), 1593-1608 (2012)

Huapaya, C. R., Arona, G. M., y Lizarralde, F.A., Enseñanza de la Ingeniería con Sistemas Tutoriales Inteligentes, doi: 10.4067/S0718-07642005000500012, Información Tecnológica, 16(5), 75-78 (2005)

Locke, J., Olsen, A., Wideman, R., Downey, M.M., Kretzmann, M., Kasari, C., y Mandell, D.S., A Tangled Web: The Challenges of Implementing an Evidence-Based Social Engagement Intervention for Children With Autism in Urban Public School Settings, doi: 10.1016/j.beth.2014.05.001, Behavior Therapy, 46, 54-67 (2014)

Manning, C.D., Raghavan, P., y Schutze, H., An Introduction to Information Retrieval, Cambridge University Press, Cambridge, Reino Unido (2009)

Mohr, D.C., Burns, M. N., Schueller, S. M., Clarke, G., y Klinkman, M., Behavioral Intervention Technologies: Evidence review and recommendations for future research in mental health, doi: 10.1016/j.genhosppsych.2013.03.008, General Hospital Psychiatry, 35(4), 332-338 (2013)

Narvaez-Romo, Y. A., Luna, G. C., Leonel, H. F., y Ruiz, J. O., Evaluación del Software Educativo Mundo Agroforestal con Jóvenes Rurales de Nariño, Colombia, doi:https://doi.org/10.4067/S0718-07642017000200015, Información Tecnológica, 28(2), 135-140 (2017)

Paul, M. J., Sarker, A., Brownstein, J. S., Nikfarjam, A., Scotch, M., Smith, K. L., y Gonzalez, G., Social Media Mining for Public Health Monitoring and Surveillance, 21st Pacific Symposium on Biocomputing, 468-479, Big Island, United States, 4 al 8 de Enero (2016)

Rana, Y., Haberer, J., Huang, H., Kambugu, A., Mukasa, B., Thirumurthy, H., Linnemayr, S., Short Message Service (SMS)-based intervention to improve treatment adherence among HIV-positive youth in Uganda: Focus group findings, doi: 10.1371/journal.pone.0125187, PLoS ONE, 10(4), 1-14 (2015)

Regneri, M., y King, D., Automatically Evaluating Atypical Language in Narratives by Children with Autistic Spectrum Disorder, doi: 10.13140/2.1.2565.3448, Natural Language Processing and Cognitive Science: Proceedings 2014,11 - 23, (2014)

Rouhizadeh, M., Computational Analysis of Language Use in Autism, Tesis Doctoral, Center for Spoken Language Understanding School of Medicine, Oregon Health y Science University, Oregon, USA (2015)

Rouhizadeh, M., Prud'hommeaux, E., Roark, B., y Santen, J. van., Distributional semantic models for the evaluation of disordered language, Proceedings of NAACL-HLT, 709 - 714, Atlanta, Georgia, 9 al 14 de Junio 2013 (2013)

Saint-Georges, C., Mahdhaoui, A., Chetouani, M., Cassel, R. S., Laznik, M. C., Apicella, F., Cohen, D., Do parents recognize autistic deviant behavior long before diagnosis? taking into account interaction using computational methods, doi: 10.1371/journal.pone.0022393, PLoS ONE, 6(7), 13 (2011)

Stephanie, D., y Julie, F., Exploring links between language and cognition in autism spectrum disorders : Complement sentences, false belief, and executive functioning, doi: 10.1016/j.jcomdis.2014.12.001, Journal of communication disorders, 54, 15-31 (2015)

Sundaram, H., Lin, Y., Choudhury, M. De, y Kelliher, A., Understanding Community Dynamics in Online Social Networks, doi: 10.1109/MSP.2011.943583, IEEE Signal Processing, 29(2), 33-40 (2012)

Tanaka, H., Sakti, S., Neubig, G., Toda, T., y Nakamura, S., Linguistic and Acoustic Features for Automatic Identification of Autism Spectrum Disorders in Children's Narrative, Proceedings of the Workshop on Computational Linguistics and Clinical Psychology: From Linguistic Signal to Clinical Reality, 88-96, Baltimore, Maryland USA (2014)

Uekermann, J., Kraemer, M., Abdel-Hamid, M., Schimmelmann, B. G., Hebebrand, J., Daum, I., ... Kis, B., Social cognition in attention-deficit hyperactivity disorder (ADHD), doi: 10.1016/j.neubiorev.2009.10.009, Neuroscience and Biobehavioral Reviews, 34(5), 734-743 (2010)

van der Aa, C., Pollmann, M., Plaat, A., y van der Gaag, R., Computer-mediated communication in adults with highfunctioning autism spectrum disorders and controls, doi: 10.1016/j.rasd.2015.11.007, Research in Autism Spectrum Disorders, 23, 15-27 (2016)

Verano, P. L. Y., El tópico perseverante en el discurso de niños diagnosticados con síndrome de asperger, Tesis de Titulación, Facultad de Letras y Ciencias Humanas, Pontificia Universidad Católica del Perú PUCP (2013)

Yamamoto, Y., y Takagi, T., Biomedical knowledge navigation by literature clustering, doi: 10.1016/j.jbi.2006.07.004, Journal of Biomedical Informatics, 40, 114-130 (2007) 
